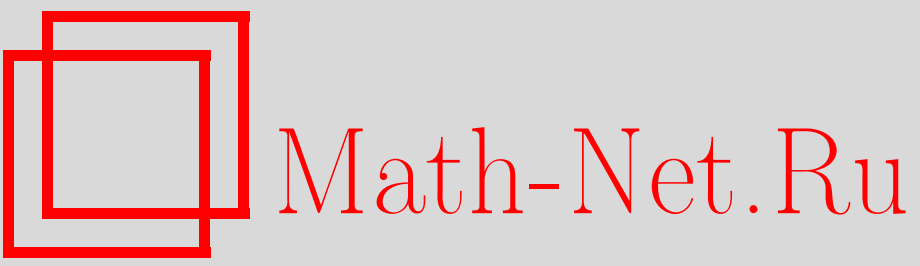

В. А. Гейлер, И. Ю. Попов, Баллистический транспорт в наноструктурах: явнорешаемые модели, ТМФ, 1996, том 107, номер 1, 12-20

DOI: https://doi.org/10.4213/tmf1134

Использование Общероссийского математического портала Math-Net.Ru подразумевает, что вы прочитали и согласны с пользовательским соглашением

http://www . mathnet.ru/rus/agreement

Параметры загрузки:

IP : 54.198 .55 .26

26 апреля 2023 г., 15:00:10 
ТЕОРЕТИЧЕСКАЯ

И МАТЕМАТИЧЕСКАЯ

ФИЗИКА

Том 107, № 1

апрель, 1996

\author{
В. А. Гейлер, И.Ю. Попов
}

\title{
БАЛЛИСТИЧЕСКИЙ ТРАНСПОРТ В НАНОСТРУКТУРАХ: ЯВНОРЕШАЕМЫЕ МОДЕЛИ
}

\begin{abstract}
$\mathrm{C}$ помошью теории самосопряженных расширений строится достаточно широкий класс явнорешаемых моделей баллистического транспорта в наноструктурах. В полученных моделях коэффициент прохождения $T(E)$ имеет простую зависимость как от параметров моделируемого устройства, так и от параметров внешнего магнитного поля. Приведены примеры моделей квантовых точек, в которых поведение $T(E)$, а следовательно, и проводимости носит осцилляционный характер.
\end{abstract}

\section{1. ВВЕДЕНИЕ}

Успехи в технологии изготовления двумерных систем носителей заряда (гетеропереходов и МОП-структур), а также структур с дополнительным латеральным конфайнментом в таких системах (квантовых каналов и квантовых точек) вызвали появление нового раздела физики конденсированного состояния: физики полупроводниковых наноструктур, называемой также мезоскопикой [1]. Сочетание чрезвычайно высокой чистоты изготовления и совершенства кристаллической структуры наноэлектронных устройств с низкой плотностью свободных носителей заряда (которой к тому же можно управлять, изменяя напряжение затвора) привело к двум принципиально важным свойствам электронного газа в обсуждаемых системах: во-первых, это большая длина свободного пробега электронов $\left(\sim 10^{-3} \mathrm{~cm}\right)$; во-вторых, относительно большая длина волны Ферми $\left(\sim 4 \cdot 10^{-6}\right.$ см). Обе эти величины сравнимы с размерами наноэлектронных устройств, и тем самым в упомянутых устройствах реализуется режим электронного баллистического транспорта, при котором рассеяние на примесях играет пренебрежимо малую роль. В силу этого стандартный подход к теории электропроводности, основанный на решении уравнения Больцмана с учетом столкновения с примесными центрами, становится неприменимым. В этом случае проводимость $\sigma$ хорошо описывается формализмом Ландауэра-Бюттикера, в рамках которого $\sigma$ непосредственно выражается через коэффициенты прохождения $\left.T(E)\right|_{E=E_{\mathrm{F}}}$ каналов наноструктуры [2-6] (здесь $E_{\mathrm{F}}-$ энергия Ферми). В простейшем случае одного канала (и одной моды) известная формула Ландауэра принимает вид [2]

$$
\sigma=\left(e^{2} / h\right) T(1-T)^{-1} .
$$

Коэффициент прохождения $T$ сушественно зависит не только от размера, но и от геометрии наноструктуры. Кроме того, в сужениях квантовых каналов сильное влияние на значение $T$ может оказывать эффект туннелирования через одиночную примесь при 
нахождении $E_{\mathrm{F}}$ вблизи резонанса Брейта-Вигнера (экспериментально это явление обнаружено в $[7,8]$ и теоретически исследовалось в [9-12] и др.). Совокупность указанных выше обстоятельств приводит к тому, что адекватным математическим аппаратом построения моделей баллистического транспорта в наноэлектронных устройствах становится теория потенциалов нулевого радиуса с внутренней структурой $[13,14]$, поскольку в рамках этой теории могут быть введены параметры, учитываюшие сушественные черты явлений переноса заряда в низкоразмерных системах. Несколько таких моделей было предложено авторами ранее [15-17]. Здесь мы предлагаем некоторую достаточно обшую схему конструирования явнорешаемых моделей наноэлектронного устройства с двумя контактами, коэффициент прохождения которого оказывается элементарно зависящим как от параметров самого устройства, так и от параметров внешних полей. По предложенной нами схеме довольно просто может быть найден, например, коэффициент прохождения изолированной квантовой точки, цепочки и периодического массива квантовых точек, мезоскопических колец и тому подобных микроэлектронных устройств (как в отсутствие, так и при наличии внешнего магнитного поля). Эту схему нетрудно обобщить на случай многотерминальных наноэлектронных устройств, на чем мы здесь подробно останавливаться не будем.

\section{2. ОСНОВНЫЕ РЕЗУЛЬТАТЫ}

Условное изображение моделируемого устройства представлено на рисунке. Гильбертово пространство "внутренних состояний" устройства обозначим $\mathcal{H}_{d}$ (например, в случае квантовой точки можно взять $\mathcal{H}_{d}=L^{2}\left(\mathbf{D}_{\boldsymbol{\varepsilon}}\right)$, где $\mathbf{D}_{\varepsilon}-$ диск в плоскости $\mathbf{R}^{2}$ достаточно малого радиуса $\left.\varepsilon\right)$. Пространство состояний каналов $\mathcal{H}_{+}=L^{2}\left(\mathbf{R}_{+}\right)$, $\mathcal{H}_{-}=L^{2}\left(\mathbf{R}_{-}\right)$. Таким образом, пространство $\mathcal{H}$ состояний системы представляет собой прямую сумму

$$
\mathcal{H}=\mathcal{H}_{-} \oplus \mathcal{H}_{d} \oplus \mathcal{H}_{+} .
$$

Если каналы заперты (т.е. если контакты $C_{ \pm}$разомкнуты), то гамильтониан системы $H^{0}$ представляет собой прямую сумму

$$
H^{0}=H_{-} \oplus H_{d} \oplus H_{+} .
$$

Здесь $H_{ \pm}$есть оператор $-d^{2} / d x^{2}$ в пространстве $L^{2}\left(\mathbf{R}_{ \pm}\right)$с условием Неймана в точке 0 (что соответствует отсутствию тока через соответствуюшие контакты $C_{ \pm}$); $H_{d}-$ гамильтониан заряженной частицы в наноэлектронном устройстве $D$. Выбор конкретного вида оператора $H_{d}$ зависит, разумеется, от свойств устройства $D$ и каждый раз является предметом специального анализа; примеры такого выбора будут приведены в разделе 3 статьи. Резольвенты операторов $H^{0}, H_{-}, H_{d}, H_{+}$обозначим через $R^{0}(\zeta)$, $R_{-}(\zeta), R_{d}(\zeta), R_{+}(\zeta)$, соответственно.

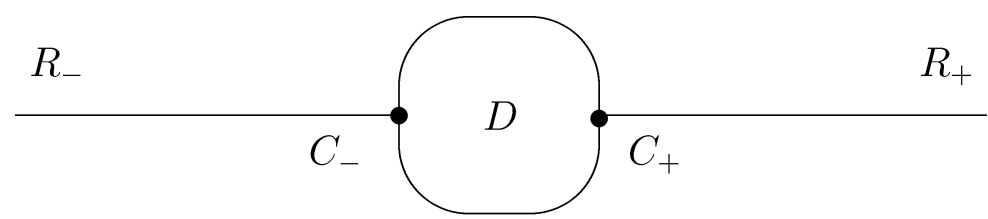

Включение контактов будем моделировать с помощью процедуры "сужения - расширения" $[13,14]$. Пусть $S_{ \pm}-$симметричный оператор, полученный сужением оператора $H_{ \pm}$на область $\mathcal{D}\left(S_{ \pm}\right)=\left\{\varphi \in \mathcal{D}\left(H_{ \pm}\right): \varphi(0)=0\right\}$. Рассмотрим также симметричный 
оператор $S_{d}$ в пространстве $\mathcal{H}_{d}$ с индексами дефекта $(2,2)$, являюшийся сужением оператора $H_{d}$. Дефектные элементы оператора $S_{d}$ будем отождествлять с контактами $C_{ \pm}$ в наноустройстве, их выбор обусловлен физической интерпретацией пространства $\mathcal{H}_{d}$ и гамильтониана $H_{d}$ и в каждом конкретном случае также является предметом специального анализа. Важно отметить, что оператор $S_{d}$ не обязательно плотно определен; в частности, так будет, если $\mathcal{H}_{d}$ - конечномерное пространство (содержательные примеры такой ситуации разобраны в $[13,14])$. Наконец, обозначим

$$
S=S_{-} \oplus S_{d} \oplus S_{+}
$$

Гамильтониан устройства с включенными контактами следует искать среди самосопряженных расширений оператора $S$ [13]. С этой целью воспользуемся формулой Крейна, описываюшей резольвенты всех самосопряженных расширений заданного симметричного оператора $S$ с помощью резольвенты некоторого его фиксированного самосопряженного расширения $[14,18,19]$. Введем для этого “стандартные” дефектные пространства [19]

$$
\mathcal{G}_{-}=\mathcal{G}_{+}=\mathbf{C}, \quad \mathcal{G}_{d}=\mathbf{C}^{2} .
$$

Контакт проводника $\mathbf{R}_{-}$с устройством осуществляется через базисные элементы $1 \in$ $\mathcal{G}_{-}$и $(1,0) \in \mathcal{G}_{d}$, соответственно. Аналогично контакт проводника $\mathbf{R}_{+}$с устройством осушествляется через базисные элементы $1 \in \mathcal{G}_{+}$и $(0,1) \in \mathcal{G}_{d}$. Обозначим через $G_{ \pm}(x, y ; \zeta)$ функцию Грина оператора $H_{ \pm}$. Тогда отображение

$$
\Gamma_{ \pm}(\zeta): \mathcal{G}_{ \pm} \rightarrow L^{2}\left(\mathbf{R}_{ \pm}\right)
$$

определяемое путем

$$
\mathbf{C} \ni \xi \mapsto G_{ \pm}(x, 0 ; \zeta) \xi \in L^{2}\left(\mathbf{R}_{ \pm}\right),
$$

представляет собой $Г$-функцию Крейна пары операторов $\left(S_{ \pm}, H_{ \pm}\right)$. Отвечающая этой паре $\mathcal{Q}$-функция Крейна $Q_{ \pm}(\zeta)$ имеет вид $Q_{ \pm}(\zeta)=G_{ \pm}(0,0 ; \zeta)$. Обозначим через $\Gamma_{d}(\zeta)$ и $Q_{d}(\zeta)$ соответственно $\Gamma$-функцию и $\mathcal{Q}$-фрункцию пары операторов $\left(S_{d}, H_{d}\right)$ :

$$
\begin{aligned}
\Gamma_{d}(\zeta): \mathbf{C}^{2} & \rightarrow \mathcal{H}_{d}, \\
Q_{d}(\zeta): \mathbf{C}^{2} & \rightarrow \mathbf{C}^{2} .
\end{aligned}
$$

Оператор $Q_{d}(\zeta)$ задается матрицей

$$
\left[\begin{array}{ll}
Q_{d}^{11}(\zeta) & Q_{d}^{12}(\zeta) \\
Q_{d}^{21}(\zeta) & Q_{d}^{22}(\zeta)
\end{array}\right]
$$

в которой $Q_{d}^{i j}(\zeta)=\overline{Q_{d}^{j i}(\zeta)}$ при вешественных $\zeta$. Введем также следуюшие обозначения для дефектных элементов оператора $S_{d}$ :

$$
\Gamma_{d}^{-}(\zeta)=\Gamma_{d}(\zeta)(1,0) ; \quad \Gamma_{d}^{+}(\zeta)=\Gamma_{d}(\zeta)(0,1)
$$

Для пары операторов $\left(S, H^{0}\right) \quad \Gamma$-функция и $\mathcal{Q}$-функция задаются прямыми суммами

$$
\begin{aligned}
\Gamma(\zeta) & =\Gamma_{-}(\zeta) \oplus \Gamma_{d}(\zeta) \oplus \Gamma_{+}(\zeta) \\
Q(\zeta) & =Q_{-}(\zeta) \oplus Q_{d}(\zeta) \oplus Q_{+}(\zeta)
\end{aligned}
$$


Как упоминалось выше, гамильтониан системы с включенными контактами мы рассматриваем как самосопряженное расширение оператора $S$. Обозначим это расширение через $H$, а его резольвенту - через $R(\zeta)$. Согласно формуле Крейна

$$
R(\zeta)=R^{0}(\zeta)-\Gamma(\zeta)[Q(\zeta)+A]^{-1} \Gamma^{*}(\bar{\zeta}),
$$

где $A$ - эрмитова $4 \times 4$-матрица (оператор в пространстве $\mathcal{G}=\mathcal{G}_{-} \oplus \mathcal{G}_{d} \oplus \mathcal{G}_{+}$), параметризующая самосопряженные расширения оператора $S$. В соответствии с изложенной выше интерпретацией дефектных элементов включение контактов означает включение "взаимодействия" между элементами $\Gamma_{-}(\zeta) \cdot 1$ и $\Gamma_{d}^{-}(\zeta)$, а также между элементами $\Gamma_{+}(\zeta) \cdot 1$ и $\Gamma_{d}^{+}(\zeta)$, соответственно. Поэтому матрицу $A$ в формуле Крейна надо выбрать в виде

$$
\left[\begin{array}{cccc}
\mu_{-} & \alpha_{-} & 0 & 0 \\
\alpha_{-} & 0 & 0 & 0 \\
0 & 0 & 0 & \alpha_{+} \\
0 & 0 & \alpha_{+} & \mu_{+}
\end{array}\right]
$$

где $\alpha_{-}$и $\alpha_{+}-$вещественные величины, характеризуюшие “идеальные" контакты $C_{ \pm}$, а вешественные же величины $\mu_{-}$и $\mu_{+}$характеризуют степень отклонения контакта $C_{-}$ (соответственно $C_{+}$) от идеального. Например, при $\mu_{+}>0$ движушаяся в проводнике $\mathbf{R}_{+}$заряженная частица испытывает отталкивание от контакта $C_{+}$, а при $\mu_{+}<0-$ притяжение. Таким образом, матрица $Q(\zeta)+A$ имеет следующую структуру:

$$
\left[\begin{array}{cccc}
\tilde{Q}_{-}(\zeta) & \alpha_{-} & 0 & 0 \\
\alpha_{-} & Q_{d}^{11}(\zeta) & Q_{d}^{12}(\zeta) & 0 \\
0 & Q_{d}^{21}(\zeta) & Q_{d}^{22}(\zeta) & \alpha_{+} \\
0 & 0 & \alpha_{+} & \tilde{Q}_{+}(\zeta)
\end{array}\right]
$$

здесь $\tilde{Q}_{ \pm}(\zeta)=Q_{ \pm}(\zeta)+\mu_{ \pm}$. Явный вид матрицы $[Q(\zeta)+A]^{-1}$ находится элементарно:

$$
[Q(\zeta)+A]^{-1}=\operatorname{det}[Q(\zeta)+A]^{-1} B,
$$

где $B$ представляет собой $4 \times 4$-матрищу, присоединенную к матрице $Q(\zeta)+A$. Простые вычисления дают

$$
\operatorname{det}(Q+A)=\tilde{Q}_{-} \tilde{Q}_{+}\left(\operatorname{det} Q_{d}\right)-\alpha_{-}^{2} \tilde{Q}_{+} Q_{d}^{22}-\alpha_{+}^{2} \tilde{Q}_{-} Q_{d}^{11}+\alpha_{-}^{2} \alpha_{+}^{2} .
$$

Выпишем также элементы матрищы $B$ :

$$
\begin{aligned}
& B_{11}=\tilde{Q}_{+}\left(\operatorname{det} Q_{d}\right)-\alpha_{+}^{2} Q_{d}^{11}, \quad B_{12}=B_{21}=\alpha_{-}\left(\alpha_{+}^{2}-\tilde{Q}_{+} Q_{d}^{22}\right), \quad B_{13}=\alpha_{-} \tilde{Q}_{+} Q_{d}^{12}, \\
& B_{14}=-\alpha_{-} \alpha_{+} Q_{d}^{12}, \quad B_{22}=\tilde{Q}_{-}\left(\tilde{Q}_{+} Q_{d}^{22}-\alpha_{+}^{2}\right), \quad B_{23}=-\tilde{Q}_{-} \tilde{Q}_{+} Q_{d}^{12}, \\
& B_{24}=\alpha_{+} \tilde{Q}_{-} Q_{d}^{12}, \quad B_{31}=\alpha_{-} \tilde{Q}_{+} Q_{d}^{21}, \quad B_{32}=-\tilde{Q}_{-} \tilde{Q}_{+} Q_{d}^{21}, \\
& B_{33}=\tilde{Q}_{+}\left(\tilde{Q}_{-} Q_{d}^{11}-\alpha_{-}^{2}\right), \quad B_{34}=B_{43}=\alpha_{+}\left(\alpha_{-}^{2}-\tilde{Q}_{-} Q_{d}^{11}\right), \quad B_{41}=-\alpha_{-} \alpha_{+} Q_{d}^{21}, \\
& B_{42}=\alpha_{+} \tilde{Q}_{-} Q_{d}^{21}, \quad B_{44}=\tilde{Q}_{-}\left(\operatorname{det} Q_{d}\right)-\alpha_{-}^{2} Q_{d}^{22} .
\end{aligned}
$$

Зафиксируем теперь значение энергии $E>0, E=k^{2}$ и рассмотрим в правом канале $\mathbf{R}_{-}$приходяшую к устройству $D$ волну $\tilde{\varphi}(x)=\exp (i k x)$. Ей соответствует решение $\varphi$ уравнения $H^{0} \varphi=E \varphi$, имеюшее вид

$$
\varphi=\left(\begin{array}{c}
\tilde{\varphi} \\
0 \\
0
\end{array}\right) .
$$


Воспользуемся "параметрическим" описанием оператора $H$ (см., например, [18]). Тогда из формулы (2) получаем, что при $\operatorname{Im} \zeta>0$ вектор $\psi$, определенньй равенством

$$
\psi=\varphi-\Gamma(\zeta)[Q(\zeta)+A]^{-1} \Gamma^{*}(\bar{\zeta})\left(H^{0}-\zeta\right) \varphi,
$$

удовлетворяет соотношению

$$
\left(H^{0}-\zeta\right) \varphi=(H-\zeta) \psi
$$

Переходя в $(3),(4)$ к пределу $\zeta \rightarrow E$, найдем решение $\psi$ уравнения $H \psi=E \psi$, являющееся суперпозицией приходящих и уходящих волн в каналах $\mathbf{R}_{ \pm}$. Для этого нам потребуется оператор $\Gamma_{-}^{*}(\bar{\zeta})$. Тривиальные вычисления показывают, что он имеет вид

$$
\Gamma_{-}^{*}(\bar{\zeta}) \varphi=\int_{\mathbf{R}_{-}} G_{-}(0, x ; \zeta) \varphi(x) d x
$$

где $\varphi \in L^{2}\left(\mathbf{R}_{-}\right)$. Следовательно, при $\operatorname{Im} \zeta>0$

$$
\Gamma_{-}^{*}(\bar{\zeta})\left(H_{-}-\zeta\right) \tilde{\varphi}=\tilde{\varphi}(0)=1,
$$

поэтому

$$
\lim _{\zeta \rightarrow E} \Gamma^{*}(\bar{\zeta})\left(H^{0}-\zeta\right) \varphi=\left(\begin{array}{l}
1 \\
0 \\
0 \\
0
\end{array}\right)
$$

и тем самым

$$
\begin{gathered}
\lim _{\zeta \rightarrow E}[Q(\zeta)+A]^{-1} \Gamma^{*}(\bar{\zeta})\left(H^{0}-\zeta\right) \varphi=\operatorname{det}[Q(\zeta)+A]^{-1}\left(\begin{array}{c}
B_{11} \\
B_{21} \\
B_{31} \\
B_{41}
\end{array}\right), \\
\lim _{\zeta \rightarrow E} \Gamma(\zeta) \operatorname{det}[Q(\zeta)+A]^{-1} \Gamma^{*}(\bar{\zeta})\left(H^{0}-\zeta\right) \varphi= \\
=\operatorname{det}[Q(E)+A]^{-1}\left(\begin{array}{c}
B_{11} G_{-}(x, 0 ; E) \\
B_{21} \Gamma_{d}^{-}(E)+B_{31} \Gamma_{d}^{+}(E) \\
B_{41} G_{+}(x, 0 ; E)
\end{array}\right) .
\end{gathered}
$$

Окончательно $\psi$ имеет вид

$$
\psi=\left(\begin{array}{c}
\tilde{\varphi} \\
0 \\
0
\end{array}\right)-[\operatorname{det}(Q(E)+A)]^{-1}\left(\begin{array}{c}
B_{11} G_{-}(x, 0 ; E) \\
B_{21} \Gamma_{d}^{-}(E)+B_{31} \Gamma_{d}^{+}(E) \\
B_{41} G_{+}(x, 0 ; E)
\end{array}\right) .
$$

По поводу формулы (5) заметим следующее. на спектре оператора $H^{0}$, поэтому символом $Q(E)$ мы обозначаем $\lim _{\zeta \rightarrow E} Q(\zeta)$, который, как будет видно из дальнейшего, сушествует во всех практически важных случаях.

Явный вид функции Грина $G_{ \pm}$хорошо известен:

$$
G_{ \pm}(x, y ; \zeta)=i(2 k)^{-1}[\exp (i k|x-y|)+\exp ( \pm i k(x+y))]
$$


где $\zeta=k^{2}, \operatorname{Im} k \geq 0$. Таким образом,

$$
G_{ \pm}(x, 0 ; E)=i k^{-1} \exp ( \pm i k x) \text { при } \quad \pm x>0 .
$$

Обозначим проекцию решения $\psi$ на пространство каналов $L^{2}\left(\mathbf{R}_{-}\right) \oplus L^{2}\left(\mathbf{R}_{+}\right)$через $\tilde{\psi}$. Из (5) и (6) получаем асимптотики

$$
\begin{aligned}
& \tilde{\psi}(x) \sim \exp (i k x)+C \exp (-i k x) \text { при } x \rightarrow-\infty, \\
& \tilde{\psi}(x) \sim-i k^{-1} \operatorname{det}[Q(\zeta)+A]^{-1} B_{41} \exp (i k x) \text { при } x \rightarrow+\infty .
\end{aligned}
$$

Тем самым для коэффициента прохождения $T(E)$ получаем следуюшую формулу, которую можно рассматривать как основной результат настоящей статьи:

$$
T(E)=\frac{\left|B_{41}\right|^{2}}{E|\operatorname{det}[Q(E)+A]|^{2}} .
$$

В большинстве практически важных случаев оператор $H_{d}$ имеет чисто точечный спектр, состоящий из собственных значений $\lambda_{n}$ конечной или бесконечной кратности. В этом случае элементы матрицы $Q_{d}(E)$ определены при всех $E, E \neq \lambda_{n}$, причем диагональные элементы вешественны при вешественных значениях $E$. Поэтому формула (7) принимает вид

$$
T(E)=\frac{E \alpha_{-}^{2} \alpha_{+}^{2}\left|Q_{d}^{21}(E)\right|^{2}}{E F_{1}^{2}(E)+F_{2}^{2}(E)},
$$

где

$$
\begin{aligned}
& F_{1}(E)=\alpha_{-}^{2} Q_{d}^{22}(E)+\alpha_{+}^{2} Q_{d}^{11}(E)+\left(\mu_{-}+\mu_{+}\right) \operatorname{det} Q_{d} \\
& F_{2}(E)=\operatorname{det} Q_{d}+E\left(\alpha_{-}^{2} \mu_{+} Q_{d}^{22}+\alpha_{+}^{2} \mu_{-} Q_{d}^{11}-\mu_{-} \mu_{+} \operatorname{det} Q_{d}-\alpha_{-}^{2} \alpha_{+}^{2}\right) .
\end{aligned}
$$

Отметим частный случай формулы $(8)$, соответствующий значениям $\mu_{ \pm}=0$ (идеальные контакты):

$$
T(E)=\frac{E \alpha_{-}^{2} \alpha_{+}^{2}\left|Q_{d}^{21}(E)\right|^{2}}{E\left(\alpha_{-}^{2} Q_{d}^{22}(E)+\alpha_{+}^{2} Q_{d}^{11}(E)\right)^{2}+\left(\operatorname{det} Q_{d}(E)-E \alpha_{-}^{2} \alpha_{+}^{2}\right)^{2}} .
$$

Формулой (9) мы ограничимся при разборе примеров в разделе 3.

\section{3. ПРИМЕРЫ}

В этом разделе мы приведем примеры применения формул (1) и (9). Пример 1 представляет собой весьма грубую модель двумерной электронной системы, находящейся в квантуюшем магнитном поле. Несмотря на некоторую примитивность в этой модели на элементарном уровне можно выявить следуюшее важное свойство систем, проявляюших целочисленное холловское квантование [20, 21]: в то время, когда холловская проводимость $\sigma_{x y}$ остается на плато, продольная проводимость $\sigma_{x x}$ обрашается в нуль. Это происходит при пиннинге уровня Ферми между уровнями Ландау (т.е. при целом числе заполнения распльвшихся уровней Ландау). Это свойство имеет место и в нашей модели, а именно, в ней $T(E)=0$, когда $E$ совпадает с одним из уровней Ландау. В соответствии с формулой Ландауэра (1) это означает обращение в нуль проводимости $\sigma_{x x}$ рассматриваемой системы, когда $E_{\mathrm{F}}$ проходит уровень Ландау. Примеры 2 и 3 на 
модельном уровне демонстрируют осцилляционный характер проводимости квантовых точек (см. обзоры [1] и [22]). Эти осцилляции связаны с изменением уровня Ферми $E_{\mathrm{F}}$, являющимся результатом изменения числа электронов в системе или изменения напряженности внешнего магнитного поля.

1. Рассмотрим двумерную электронную систему, находящуюся в однородном квантуюшем магнитном поле напряженности $\vec{B}$, перпендикулярном ее плоскости. Пренебрегая конечностью размеров системы, отождествим ее с плоскостью $\mathbf{R}^{2}$. Контакты расположим симметрично относительно точки $(0,0)$ в точках $\vec{r}_{1}=(-a, 0)$ и $\vec{r}_{2}=(a, 0)$. В рассматриваемом случае оператор $H_{d}$ представляет собой оператор Ландау, т.е. самосопряженный оператор в $L^{2}\left(\mathbf{R}^{2}\right)$, определяемый следующим дифференциальным выражением:

$$
\left(-\hbar^{2} / 2 m\right)\left[\left(\partial / \partial x_{1}+i \pi \xi x_{2}\right)^{2}+\left(\partial / \partial x_{2}-i \pi \xi x_{1}\right)^{2}\right]
$$

где $\xi$ - число квантов потока магнитного поля через единичную плошадку плоскости $\mathbf{R}^{2}$ (см., например, [23]). Спектр этого оператора чисто точечный и состоит из бесконечно вырож денных уровней Ландау - собственных чисел

$$
\varepsilon_{l}=(l+1 / 2) \hbar \omega_{c}, \quad l=0,1, \ldots,
$$

где $\omega_{c}=|e B| / c m$ - циклотронная частота. В этом случае элементы матрищы $Q_{d}(E)$ известны [23, 24], а именно,

$$
Q_{d}^{11}(E)=Q_{d}^{22}(E)=-\left(m / 2 \pi \hbar^{2}\right)\left[\psi\left(1 / 2-E / \hbar \omega_{c}\right)+\log \left(\pi|\xi| a^{2}\right)+2 C_{\mathrm{E}}\right]
$$

В формуле (11) $\psi(x)$ - логарифмическая производная Г-функции Эйлера, $C_{\mathrm{E}}-$ постоянная Эйлера. Для внедиагональных элементов матрицы $Q_{d}$ имеем $Q_{d}^{j k}(E)=$ $G_{d}\left(\vec{r}_{j}, \vec{r}_{k} ; E\right)$, где $G_{d}$ - функция Грина оператора $H_{d}$, которая имеет вид

$$
\begin{aligned}
G_{d}\left(\vec{r}, \vec{r}^{\prime} ; E\right)= & \left(m / 2 \pi \hbar^{2}\right) \Gamma\left(1 / 2-E / \hbar \omega_{c}\right) \exp \left[-\pi i \xi \vec{r} \wedge \vec{r}^{\prime}-\pi|\xi|\left(\vec{r}-\vec{r}^{\prime}\right)^{2} / 2\right] \times \\
& \times \Psi\left(1 / 2-E / \hbar \omega_{c}, 1 ; \pi|\xi|\left(\vec{r}-\vec{r}^{\prime}\right)^{2}\right)
\end{aligned}
$$

где $\Psi(a, c ; x)$ - вырожденная гипергеометрическая функция. Тем самым

$$
\begin{aligned}
Q_{d}^{12}(E)=Q_{d}^{21}(E)= & \left(m / 2 \pi \hbar^{2}\right) \Gamma\left(1 / 2-E / \hbar \omega_{c}\right) \times \\
& \times \exp \left[-2 \pi|\xi| a^{2}\right] \Psi\left(1 / 2-E / \hbar \omega_{c}, 1 ; 4 \pi|\xi| a^{2}\right) .
\end{aligned}
$$

Формулы (11)-(13) показывают, что при $E=\varepsilon_{l}$ функции $Q_{d}^{11}(E)$ и $Q_{d}^{12}(E)$ имеют полюсы с разными вычетами (за исключением специально подобранных значений $a$, которыми мы по понятным причинам пренебрегаем). Тем самым числитель в (9) имеет полюс второго порядка, а знаменатель - четвертого. Отсюда вытекает, что $T(E)=0$, как только $E=\varepsilon_{l}$.

2. Рассмотрим теперь коэффициент прохождения через квантовую точку произвольной формы. В этом случае $\mathcal{H}_{d}=L^{2}(\Omega)$, где $\Omega$ - ограниченная область в $\mathbf{R}^{2}$ с кусочно-гладкой границей, а оператор $H_{d}$ представляет собой лапласиан $-\Delta$ с условием Неймана на границе $\partial \Omega$. Обозначим через $\left(\lambda_{n}\right)_{n \geq 1}$ последовательность всех собственных чисел задачи Неймана для области $\Omega$, занумерованных с учетом кратности, а через $\left(\varphi_{n}\right)$ - соответствующую ортонормальную последовательность собственных функций. Зафиксируем число $\varepsilon_{0}, \varepsilon_{0}<0$, и пусть $a_{1}\left(\right.$ соответственно $\left.a_{2}\right)$ - положение контакта $C_{-}$ 
(соответственно $C_{+}$) в области $\Omega$. Следуя $[15,16,25]$, мы приходим к таким значениям элементов матрицы $Q_{d}(E)$ при $E \geq 0, E \neq \lambda_{n}$ :

$$
\begin{aligned}
& Q_{d}^{j j}(E)=\left(E-\varepsilon_{0}\right) \sum_{n=1}^{\infty}\left|\varphi_{n}\left(a_{j}\right)\right|^{2}\left(\lambda_{n}-E\right)^{-1}\left(\lambda_{n}-\varepsilon_{0}\right)^{-1}, \quad j=1,2, \\
& Q_{d}^{j k}(E)=\sum_{n=1}^{\infty} \bar{\varphi}_{n}\left(a_{j}\right) \varphi\left(a_{k}\right)\left(\lambda_{n}-E\right)^{-1}, \quad j \neq k .
\end{aligned}
$$

Как и в примере 1 , формулы $(14),(15)$ приводят к осцилляциям величины $T(E)$, которая обрашается в нуль при прохождении уровня Ферми $E_{\mathrm{F}}$ через значения энергии $\lambda_{n}$.

3. Кратко обсудим особенности коэффициента прохождения через квантовую точку, находяшуюся в магнитном поле. В этом случае можно взять $\mathcal{H}_{d}=L^{2}(\Omega)$, где $\Omega$ - как в примере 2 , а в качестве $H_{d}$ взять оператор вида $(11)$ с условиями Неймана на $\partial \Omega$. Затем можно воспользоваться формулами (14), (15). В этом случае основной интерес представляет зависимость $T(E)$ от напряженности магнитного поля $B$. Для этого достаточно знать зависимость от $B$ собственных чисел $\lambda_{n}$. Поскольку в общем случае задача нахождения аналитического вида этой зависимости не разрешима, в теоретических исследованиях транспортных свойств квантовых точек в магнитном поле применяют модель, в которой потенциалом конфайнмента квантовой точки служит параболический потенциал $V\left(x_{1}, x_{2}\right)=\left(m \omega^{2}\right)\left(x_{1}^{2}+x_{2}^{2}\right) / 2$. Здесь $\omega=\hbar / m L^{2}$, где $L$ - характерный размер квантовой точки. В этом случае $H_{d}=H_{\mathrm{L}}+V$, где $H_{\mathrm{L}}$ - гамильтониан Ландау, т.е. самосопряженный оператор в $L^{2}\left(\mathbf{R}^{2}\right)$, определенный дифференциальным выражением (10). Собственные числа $\lambda_{n}$ оператора $H_{d}$ представляют собой уровни Фока-Дарвина

$$
\varepsilon_{m n}(B)=(m+1 / 2) \hbar \omega_{-}+(n+1 / 2) \hbar \omega_{+}, \quad m, n=0,1, \ldots,
$$

где $\omega_{ \pm}=\left(\sqrt{\omega_{c}^{2}+4 \omega^{2}} \pm \omega_{c}\right) / 2[22]$. Расположение точек $\varepsilon_{m n}(B)$ на полуоси $\mathbf{R}_{+}$регулярно, если отношение $\omega_{-} / \omega_{+}$рационально, и иррегулярно в противном случае. В соответствии с этим зависимость $T$ от напряженности поля $B$ носит "хаотический” характер (см. $[1,22]$ и др.).

По поводу разобранных примеров необходимо сделать одно замечание. Уровень Ферми $E_{\mathrm{F}}$ в нашей модели определяется полным гамильтонианом $H$, поэтому он, вообше говоря, не совпадает с собственными числами оператора $H_{d}$. Тем самым в рассмотренных нами примерах проводимость не обрашается тождественно в нуль.

В заключение авторы благодарят В.А.Маргулиса за обсуждение результатов настоящей статьи. За финансовую поддержку мы признательны РФФИ (гранты № 95-01-00439 и 95-02-04871) и ГК РФ по высшему образованию (грант № 94-2.7-1067).

\section{Список литературы}

[1] Beenakker C.W.J., van Houten H. // Solid State Phys. V. 44 / Eds. H.Ehrenreich, D.Turnbull. New York: Academic Press, 1991. P. 1.

[2] Landauer R. // IBM J. Res. Dev. 1957. V. 1. P. 223.

[3] Landauer R. // Phil. Mag. 1970. V. 21. P. 863.

[4] Büttiker M. // Phys. Rev. Lett. 1986. V. 57. P. 1761.

[5] Büttiker $M$. // Electronic properties of multilayers and low-dimensional semiconductor structures / Eds. Chamberlain J. et al.. New York: Plenum Press, 1990. P. 51.

[6] Büttiker M. // J. Phys. Condens. Matter. 1993. V. 5. P. 9369. 
[7] McEuen P.L., Alphenaar B.W., Wheeler R.G. et al. // Surf. Sci. 1990. V. 229. P. 312.

[8] Eugster C.C. et al. // Phys. Rev. B. 1992. V. 46. P. 10146.

[9] Левинсон Я.Б., Лубин М.И., Сухоруков Е.В. // Письма в ЖЭТФ. 1991. Т. 54. С. 405.

[10] Levinson Y.B., Lubin M.I., Sukhorukov E.V. // Phys. Rev. B. 1992. V. 45. P. 11936.

[11] Geyler V.A. // Proc. 2nd Int. Conf. Nanometer Scale Sci. and Technol. Part 3. Moscow, 1994. P. 834.

[12] Гейлер В.А., Маргулис В.А., Чучаев И.И. // Письма в ЖЭТФ. 1993. Т. 58. С. 668.

[13] Павлов Б. // ТМФ. 1984. Т. 59. № 3. С. 345-353.

[14] Павлов Б. // УМН. 1987. Т. 42. Вып. 6 С. 99.

[15] Попов И.Ю. // ФТТ. 1994. Т. 36. С. 1918.

[16] Попов И.Ю., Попова С.Л. // ЖТФ. 1994. Т. 64. № 125 1. С. 23.

[17] Geyler V.A., Popov I.Yu. // Phys. Lett. A. 1994. V. 187. P. 410.

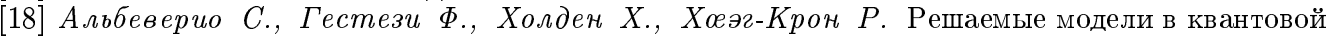
механике. М.: Мир, 1991.

[19] Крейн М.Г., Лангер Г.К. // Функц. анализ и его прилож. 1971. Т. 5. № 125 2. С. 59.

[20] Квантовый эффект Холла. Сб. статей / Сост. А.Я.Шик и Ю.В.Шмарцев. М.: Мир, 1986.

[21] Квантовый эффект Холла / Ред. Р. Прендж и С. Гирвин. М.: Мир, 1989.

[22] Chakraborty T. // Comments Cond. Matter Phys. 1992. V. B16. P. 35.

[23] Гейлер В.А. // Алгебра и анализ. 1989. Т. 3. № 3. С. 1.

[24] Гейлер В.А., Маргулис В.А. // ТМФ. 1984. Т. 58. №3. С. 461-472.

[25] Киселев А.А., Павлов Б. // ТМФ. 1994. Т. 100. № 3. С. 354-366.

Мордовский государственный университет (Саранск), Санкт-Петербургский институт

Поступила в редакцию точной механики и оптики

16.VI.1996 г

\section{V.A. Geyler, I. Yu. Popov \\ BALLISTIC TRANSPORT IN NANOSTRUCTURES: EXPLICITLY SOLVABLE MODELS}

A relatively wide class of explicitly solvable models of the ballistic transport in nanostructures is constructed by means of operator extension theory. In the obtained models the transmission coefficient $T(E)$ has a simple dependence on the modeled device parameters as well as on the parameters of an external magnetic field. Some exemples of quantum point models are given, in which $T(E)$, and hence the conductance, exhibits an oscillatory behaviour. 